The Chinese Society of Theoretical and Applied Mechanics

Chinese Journal of Mechanics Press, Beijing, China

Allerton Press, INC., New York, U.S.A.

\title{
MICROGRAVITY EXPERIMENTS OF TWO-PHASE FLOW PATTERNS ABOARD MIR SPACE STATION*
}

\author{
Zhao Jianfu (赵建福) Xie Jingchang (解京昌) Lin Hai (林 海) Hu Wenrui (胡文瑞) \\ (NMLC, Institute of Mechanics, Chinese Academy of Sciences, Beijing 100080, China) \\ A.V. Ivanov A.Yu. Belyaev \\ (Keldysh Research Center, Russian Aviation and Space Agency, Moscow 125438, Russia)
}

\begin{abstract}
A first experimental study on two-phase flow patterns at a long-term, steady microgravity condition was conducted on board the Russian Space Station "MIR" in August 1999. Carbogal and air are used as the liquid and the gas phase, respectively. Bubble, slug, slug-annular transitional, and annular flows are observed. A new region of annular flow with lower liquid superficial velocity is discovered, and the region of the slug-annular transitional flow is wider than that observed by experiments on board the parabolic aircraft. The main patterns are bubble, slugannular transitional and annular flows based on the experiments on board MIR space station. Some influences on the two-phase flow patterns in the present experiments are discussed.
\end{abstract}

KEY WORDS: two-phase flow, microgravity fluid physics, space experiment

\section{INTRODUCTION}

Two-phase gas-liquid flow has wide applications both on Earth and in space. It occurs in a variety of process equipment on Earth, such as petroleum production facilities, condensers and reboilers, power systems and core cooling of nuclear power plants during some emergency operation. The space applications include active thermal control systems, power cycles, storage and transfer of cryogenic fluids, and so on ${ }^{[1]}$. However, our present knowledge on the flow patterns, pressure drops, and heat transfer of two-phase flows, which is built on a foundation of a large empirical database developed with the aid of numerous meticulous experiments under the terrestrial environment, is far from complete. It is believed that the gravity is a dominant factor for the complication of two-phase flows. Then at microgravity conditions, due to the weakening or removal of the gravity, two-phase flows will be essentially much simpler than those at the normal gravity. Therefore, the investigations of two-phase flows at low and microgravity will be conductive to revealing of the mechanism underlying the flow pattern transitions, and then developing of more mechanistic models for the flow pattern transitions of two-phase flows both on Earth and in space.

Received 14 July 2000

* The project supported by the National Natural Science Foundation of China (19789201), the Ministry of Science and Technology of China (95-Yu-34), and the Post-doctoral Science Foundation of China 
In the past 15 years or so, some experimental investigations of the two-phase flows have been conducted in reduced gravity conditions aboard an aircraft flying parabolic trajectories or free falls in a drop tower. However, the limited test period associated with this method makes it difficult to eliminate the transient effect of the rapid change in gravitational fields. Some other creative ways of simulating microgravity in a laboratory environment, such as the use of small inside diameter tubes and density-matched immiscible liquids, were also used to increase the impact of surface tension force and reduce that of gravity. These methods can create gravity-independent two-phase flows with some characteristics of two-phase flows at a reduced gravity. The disadvantage, however, is that they are not complete simulations of microgravity two-phase gas-liquid flows. Efforts have also been made to develop the criteria of the flow pattern transitions at a reduced gravity. A comprehensive review of the different methods of testing and modelling of the flow pattern transitions at reduced gravity can be found in $\mathrm{Zhao}^{[1]}$.

A series of two-phase flow experiments were performed on board the Russian Space Station "MIR" in August 1999. The microgravity condition of the background environment of the space station is no more than $10^{-5} g$ (where $g$ denotes the acceleration of gravity on the ground). The long-term, steady reduced gravity condition can avoid the disadvantages mertioned above, and then a better understanding of the characteristics of two-phase flows at the microgravity condition may be obtained.

\section{EXPERIMENTAL FACILITIES}

The test facility, mounting in the "Volna-2A" facility on board the Russian Space Station "MIR", is shown schematically in Fig.1. Certain volume of air and carbogal, which are, respectively, used as the experimental fluids of gas and liquid phases, are operated in a close-loop system. Carbogal is an odourless, colourless, and non-toxic liquid. Its main characteristics are as follows: density $1858 \mathrm{~kg} / \mathrm{m}^{3}$, viscosity $1.05 \times 10^{-6} \mathrm{~m}^{2} / \mathrm{s}$, surface tension $0.019 \mathrm{~N} / \mathrm{m}$.



Fig.1 Schematic of the experimental facility aboard the Russian Space Station "MIR"

The $10 \mathrm{~mm}$-bore circular test tube is drilled in an organic glass prism with a $16 \times 16 \mathrm{~mm}^{2}$ square cross section. The contact angle of this gas-liquid-solid system is in the range of $1^{\circ} \sim 7^{\circ}$, which indicates that the liquid will well wet the wall of the test tube. The length 
of the test tube is $356 \mathrm{~mm}$, which is limited by the spatial restrictions of the space station. The two phases are supplied to the test tube through a Venturi mixer specially designed to weaken the entrance effects. Three groups of $0.75 \mathrm{~mm}$ diameter drillings, six drillings per group, are located at different cross-sections but uniformly around the periphery of the Venturi tube. Carbogal is injected axially while air is introduced peripherally. After the test tube, the gas-liquid mixture is separated in a separator. Carbogal remains in the separator and air fills in a rubber vessel. After the separator is totally filled with carbogal or the decreased air pressure in the receiver falls down to a specific value, carbogal is pumped from the separator to the storage vessel while air is compressed from the rubber vessel to the receiver. Further experimental runs can be conducted again.

Two turbine flowmeters with different nominal flow ranges are used for the measurement of the liquid flow rate in the space experiments. The frequency output is shown on the display screen of a multimeter. They were calibrated on Earth prior to their delivery to the space station. The averaged gas flow rate is determined through the pressure drop in the receiver over the measurement period, using the ideal gas state equation. It was shown in the calibration on Earth that the absolute pressure in the test tube is approximately equal to the atmospheric pressure when the rubber vessel is not full, and that the change of the temperature in the receiver can be neglected for determining the used air amount. Generally, the uncertainties in the measurement of the gas and liquid superficial velocities are estimated to be less than $10 \%$ over the ranges investigated in these studies.

The flow patterns in the second half part of the test tube (longer than $150 \mathrm{~mm}$ ) are recorded by a digital video camera SONY DCR-VX1000. The information of the gas and liquid flow rates is also recorded by the video camera and then the gas and liquid superficial velocities can be calculated based on the replay of the videotape in the laboratory on Earth. Unfortunately, due to the incorrect operation, the videotape recording was conducted with the automatic mode of exposure and sharpness during the space experiment, instead of the exposure time of $1 / 2000$ second as designed. That results in blur images of the phase distribution in the test tube, and then some difficulties arise in the identification of the characteristics of the two-phase flow patterns, particularly for the cases of higher flow rates.

\section{EXPERIMENTAL RESULTS}

There are 42 experimental runs performed at the microgravity condition. The gas superficial velocity $\left(U_{\mathrm{SG}}\right)$ and the liquid superficial velocity $\left(U_{\mathrm{SL}}\right)$ are varied in the range of $0.09 \sim 6.29 \mathrm{~m} / \mathrm{s}$ and $0.001 \sim 0.81 \mathrm{~m} / \mathrm{s}$, respectively. The information about the flow structure is obtained by carefully observing a sequence of successive frames with replay of the videotape in the laboratory. Some representative hand traces are displayed in Fig. 2 to convey the basic observations of the flow patterns at microgravity.

In accordance with the accepted terminology, the flow patterns obtained at microgravity can be classified as four kinds:

(1) Bubble flow. In the bubble flow regime, the size of gas bubbles is smaller than the tube diameter. Bubbles with different diameters move at approximately the same velocity, showing that the local velocity slip between the two phases is small at microgravity.

(2) Slug flow. Elongated gas bubbles (so-called Taylor bubbles) having regular hemispherical nose shapes occur in the test tube. The flow structure is essentially axisymmetrical. 


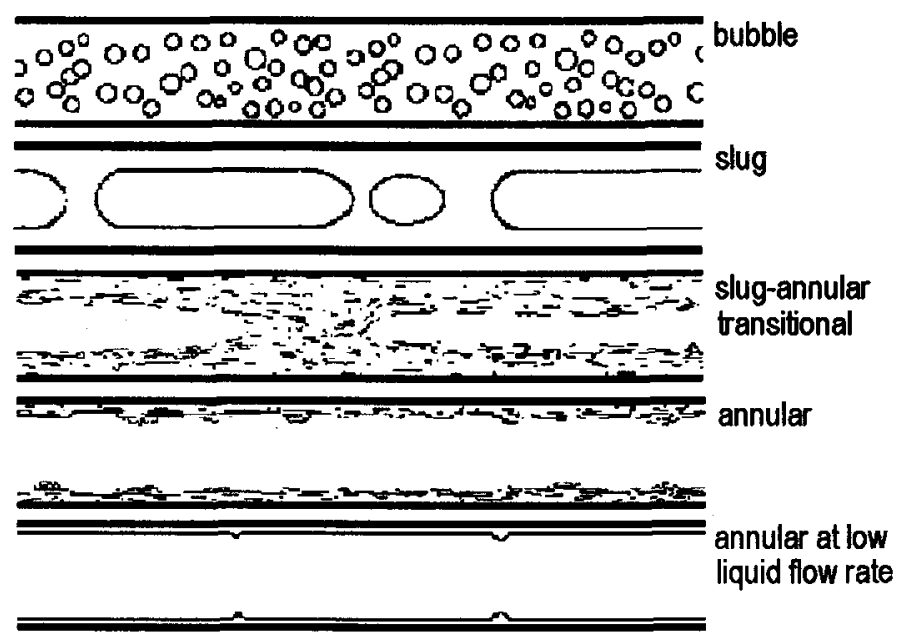

Fig.2 Flow patterns observed at microgravity condition

Length of Taylor bubbles does not exceed the observation section. For the Taylor bubble with length of $3 \sim 4$ diameters of the test tube, not only the nose but also the rear has a shape close to hemisphere. Shorter bubble has a slightly pronounced conical shape along, while the rear of longer Taylor bubbles may lose the spherical shape.

(3) Slug-annular transitional flow. It is transitional flow between slug and annular ones, in which the liquid flows as a film continuously along the wall of the test tube as in the annular flow. However, the liquid plug flows irregularly in the gaseous core, and then gas slugs are formed as in the slug flow. In contrast to the slug flow, the formed gas slugs always have much irregular shapes.

(4) Annular flow. It is observed when the gas phase flows uninterruptedly at the center of the test tube and the liquid phase flows in a wavy film at the wall.

There are also 7 preliminary runs conducted at the microgravity condition, in which the liquid flow rates are substantially lower than the flowmeter sensitivity threshold and then no signal but zero is shown on the indicating display of the multimeter. The liquid velocities, however, can be evaluated in the range of $0.001 \sim 0.013 \mathrm{~m} / \mathrm{s}$ based on analysis of the roll wave propagation velocity in the liquid film. These flows are clearly identified as the annular flow with a much thin liquid film. In two of these runs with lower gas flow rates, rupture of the liquid film occurs and the formed liquid-bridge moves quickly downwards. The film is remarkably smooth except at the wave crest. The features observed in these preliminary runs are similar with the liquid-liquid two-phase flow in a glass capillary tube of inside diameter $54 \mu \mathrm{m}$ reported by Aul \& Olbricht ${ }^{[2]}$.

According to the above observations, a flow pattern map is presented in Fig.3. It is obvious that there exists a new region of annular flow that was not observed before. This region covers a relatively wide range of the gas superficial velocity at much low liquid superficial velocities. A simple interpretation of the existence of this new regime of annular flow can be made based on the excellent wettability of the liquid phase. However, the detailed criterion of its existence is still an open question. 


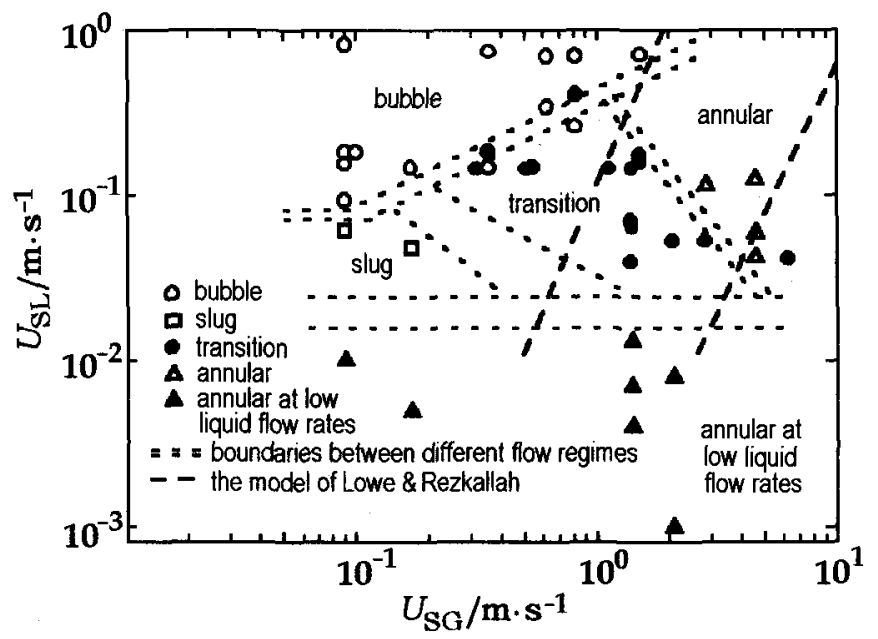

Fig.3 Microgravity two-phase flow pattern map

\section{DISCUSSIONS}

Comparing with the flow pattern maps obtained before, for example, the flow pattern map of Lowe \& Rezkallah ${ }^{[3]}$, another obvious feature of the present flow pattern map is the extension of the region of the slug-annular transitional flow and the reduction of the region of the slug flow. Based on the present experiments, the flow patterns of the two-phase flow in microgravity are mainly the bubble flow, the annular flow, and the slug-annular transitional flow, and the slug flow occupies only a small region in the $U_{\mathrm{SG}}-U_{\mathrm{SL}}$ diagram. It seems that further investigation on the transitional region is necessary to verify whether the slug-annular transitional flow is one of the fundamental patterns in the two-phase flows at a persistent microgravity environment.

The length-to-diameter ratio in the present experiment is nearly 36 , which is limited by the spatial restrictions of the space station. The flow patterns transition model of Lee ${ }^{[4]}$ is the one in the literature in which the entrance influences on the flow patterns are taken into account. Comparison between the experimental data and the predictions of the Lee's model can give information of the entrance influence on the flow patterns. However, the main patterns in Lee's model are the bubble flow, the slug flow and the annular flow, which does not agree with the observations in the present space experiments. Then the slug-annular transitional flow observed in the present experiments is regarded as an intermittent flow pattern like the slug flow.

The transition criteria of Lee's model are derived from the scaling analysis of related forces in the formation of bubbles at the gas injector at the inlet of the tube. Of them, the criteria involved in the present case are given and discussed as follows.

The gas flow rates are subdivided into three domains of "low", "middle" and "high" gas flow rates based on the ratio of the gas kinetic force and the surface tension at the gas injector. Different mechanisms will control the flow pattern transitions in the different domains. The boundaries between each two domains lie, respectively, in

$$
\frac{\rho_{\mathrm{G}}\left(U_{\mathrm{SG}} A_{t} / n\right)^{2} / A_{n}}{\pi d_{n} \sigma f(\varphi)}=0.1 \text { or } 10
$$

where $\rho, \sigma$ are, respectively, the density and the surface tension, $A_{t}$ is the tube cross-section 
area, $A_{n}, n$ and $d_{n}$ are, respectively, the cross-section area, the number and the diameter of gas injector nozzles, $f(\varphi)$ is a function of the inclined angle between the nozzle and the tube. The subscripts $\mathrm{G}$ and $\mathrm{L}$ denote the gas- and liquid-phase. According to $\mathrm{Kim}^{[5]}$, the value of $f(\varphi)=0.9$ is taken for the cross-flow multi-nozzle system used in the present study.

In the "low" gas flow rate domain, the gas kinetic force is too weak to overcome the surface tension, which prevents the departure of gas bubbles from the nozzle of the gas injectors. Since the liquid velocity is greater than the gas velocity coming out of the nozzle in this case, the drag force is the sole positive force for the bubble departure. There would be no strong bubble coalescence near the nozzle exit and then the flow structure might depend on the bubble size near the nozzle, which is controlled by the balance of the drag force and the surface tension. Based on the above argument, the bubble-to-slug transition will occur at

$$
U_{\mathrm{SL}}=\sqrt{\frac{8 \sigma d_{n}}{C_{\mathrm{D}} \rho_{\mathrm{L}} D^{2}}}
$$

where $C_{\mathrm{D}}$ is the drag coefficient, $D$ is the inner diameter of the test tabe. According to Maxworthy et al. ${ }^{[6]}$, the curve of the drag coefficient of a gas bubble against the bubble Reynolds number $R e_{\mathrm{B}}=U_{\mathrm{B}} D_{\mathrm{B}} / \nu_{\mathrm{L}}$ may be also dependent upon the Morton number $M o=$ $g \nu_{\mathrm{L}}^{4} \rho_{\mathrm{L}}^{3} / \sigma^{3}$, where $U_{\mathrm{B}}, D_{\mathrm{B}}$ denote the velocity and diameter of the gas bubble, and $\nu_{\mathrm{L}}$ denotes the liquid viscosity. In the present experiment the Morton number has a value of $M o \approx$ $10^{-13}$. Assuming that at the bubble-to-slug transition the size of the gas bubble formed near the gas injector is approximated as the diameter of the throat of the Venturi mixer $(5.3 \mathrm{~mm})$. The relative velocity between the gas bubble and liquid flow in the "low" gas flow rate domain may have an order of $0.1 \mathrm{~m} / \mathrm{s}$ as that of the liquid superficial velocity. Therefore, the bubble Reynolds number is approximated as 500 , and the value of $C_{\mathrm{D}} \approx 0.135$ will be taken $^{[6]}$.

In the "high" gas flow rate domain, when the gas velocity coming out of the nozzle is much far greater than the liquid velocity, namely

$$
\frac{U_{\mathrm{SL}}}{U_{\mathrm{SG}} / n} \ll \frac{A_{t}}{A_{n}}
$$

the gas kinetic force is sufficient to push the gas bubble forward before it becomes large and/or departs from the nozzle. The jet of the gas phase is expected to extend over a substantial length in the tube, and the existence of an annular flow structure is thus possible. For the case of a multi-nozzle injector, the merger of the multi-jet flow from the nozzles would also result in an annular flow structure. Although the smooth gas-liquid annular flow is unstable according to the linear stability theory, an annular flow structure with a strongly wavy gas-liquid interface would be maintained if the effective eddy viscosity in the turbulent gas flow is larger than that of the liquid at wall ${ }^{[7]}$.

In the "middle" gas flow rate domain, when

$$
\frac{U_{\mathrm{SG}} / n}{U_{\mathrm{SL}}} \leq 0.025 C_{\mathrm{D}} \frac{\rho_{\mathrm{L}}}{\rho_{\mathrm{G}}} \frac{A_{n}}{A_{t}}
$$

the added mass force due to the bubble expansion in the liquid phase would be negligible. Based on the balance of the gas kinetic force and the drag force, the bubble size might be determined approximately as

$$
D_{\mathrm{B}} / D=K a \sqrt{\left(U_{\mathrm{SG}} / n\right) / U_{\mathrm{SL}}}
$$


where $K a$ is an empirical constant with the baseline value of 0.4 . In this case, the surface tension counteracts the effect of the dynamic pressure due to the turbulent fluctuation in the liquid phase. If the liquid superficial Weber number

$$
W e_{\mathrm{SL}}=\rho_{\mathrm{L}} U_{\mathrm{SL}}^{2} D / \sigma \leq 870 \sqrt{U_{\mathrm{SL}} /\left(U_{\mathrm{SG}} / n\right)} R e_{\mathrm{SL}}^{1 / 5}
$$

where the liquid superficial Reynolds number $R e_{\mathrm{SL}}=U_{\mathrm{SL}} D / \nu_{\mathrm{L}}$, the dynamic pressure due to the turbulent fluctuation in the liquid phase can not overcome the surface tension, and then the bubble break-up process will not occur. On the other hand, bubble coalescence always occurs in the present domain. Therefore, according to Eq.5, bubble flow will occur only when

$$
\sqrt{U_{\mathrm{SL}} /\left(U_{\mathrm{SG}} / n\right)}=C \gg K a
$$

Here a value of $C=6 K a=2.4$ is taken in the present study. The arbitrary selection of the value of $C$ may dramatically affect the position of the transition boundary, and weaken the entrance influence, i.e. the effect of the number of the nozzles.

It is clearly shown in Fig.4 that the predictions of the Lee's model agree with the experimental observations if the slug-annular flow is also regarded as an intermittent flow as the slug flow. It may indicate that these flows probably do not reach the last state and still in the development due to the relative short length-to-diameter ratio of the test tube used in the present study. However, it ought to be noted here that Eq.7 with the present value of $C$ is completely identical to the empirical model of Jayawadena et al. ${ }^{[8]}$. The later is similar with the widely used drift-flux model for predicting the bubble-to-slug transition in the fully developed two-phase flows at microgravity ${ }^{[9]}$. Thus the developing length in the "middle" gas flow rate domain might be much small, and the flow structures observed in the present study might be fully developed.

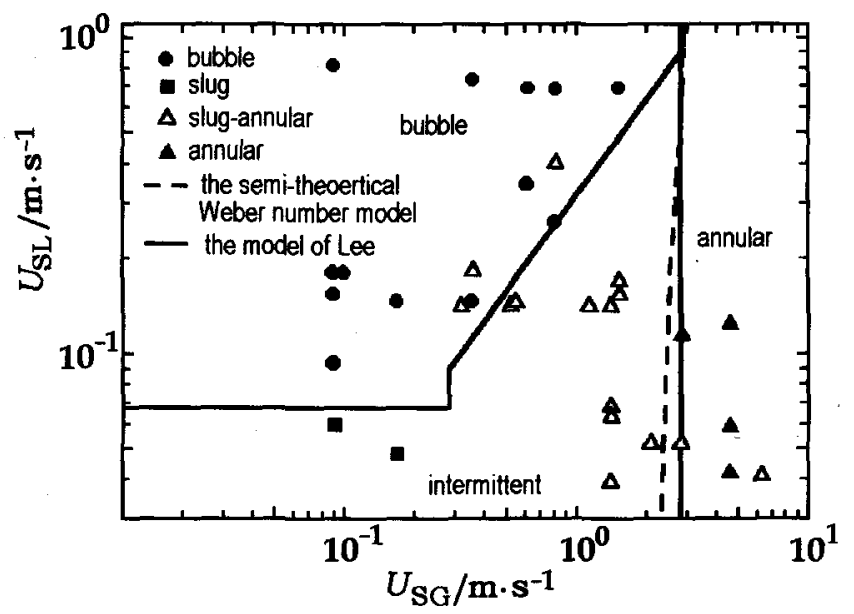

Fig.4 Comparison between the experimental data and the predictions of the model of Lee ${ }^{[4]}$ and the semi-theoretical Weber number model ${ }^{[10]}$

For predicting the slug-to-annular transition in fully developed two-phase flows at the microgravity condition, a semi-theoretical Weber number model was recently proposed by Zhao \& $\mathrm{Hu}^{[10]}$. It is assumed in the model that the basic mechanism controlling the transition 
from slug to ordinary annular flows is the balance between the impulsive force due to the gas inertia and the surface tension force. With the use of the drift-flux relationship, the transition can be expressed in dimensional form as

$$
\begin{aligned}
& U_{\mathrm{SG}}=\sqrt{\frac{4 \kappa C_{0} \sigma \varepsilon^{3 / 2}(1-\varepsilon)}{\left(C_{0}-1\right) \rho_{\mathrm{G}} D}} \\
& U_{\mathrm{SL}}=\frac{1-C_{0} \varepsilon}{C_{0} \varepsilon} U_{\mathrm{SG}}
\end{aligned}
$$

where $\varepsilon$ is the gas void fraction, $C_{0}$ is the gas phase distribution parameter and $\kappa$ is an empirical parameter of an order of 1 . The value of $\kappa=1$ is taken in the above formulae, while $C_{0}=1.16$ is taken for the two-phase flow in a circular tube at microgravity, as suggested by Zhao \& $\mathrm{Hu}^{[10]}$.

It is shown in Fig.4 that the transition from slug to ordinary annular flow is also predicted with good satisfaction by the new Weber number model, which is very close to the prediction of Lee's model. It may indicate that the entrance influence can also be negligible for such flows.

\section{CONCLUSION}

The first two-phase gas-liquid flow experiments at a long-term, steady microgravity condition have been conducted on board the Russian Space Station "MIR". A new region of annular flow with lower liquid superficial velocity is discovered firstly. It is found in the present experiment that the region of the slug-annular transitional flow is wider than that observed before using some ground-based technology such as parabolic aircraft and that the region of slug flow is reduced dramatically. Based on the present experiments, the basic flow patterns of two-phase flow at microgravity environment are the bubble, annular and slug-annular transitional flows.

The entrance influence on the flow patterns is also discussed by comparing the experimental data with the predictions of the flow pattern transition models. The Lee's model ${ }^{[4]}$ is the sole one in the literature that takes into account the effects of inlet geometry. The predictions agree well with the experimental data. However, the predictions of Lee's model for the "middle" and "high" gas flow rates are very close to those of the widely used models [8 10] for predicting the flow pattern transitions in fully developed two-phase flows at the microgravity condition. It indicates that the developing length in the "middle" and "high" gas flow rate domains may be much small, and the flow structures observed in the present study may be fully developed. Due to the small length-to-diameter ratio of the test tube limited by the spatial restrictions of the space station, the two-phase flows for the "small" gas flow rates may be still in the development.

Acknowledgement - The space experiment was performed by the collaboration with the Keldysh Research Center of Russia. The authors wish to express their gratitude to the cosmonauts for their tremendous efforts.

\section{REFERENCES}

1 Zhao JF. A review of two-phase gas-liquid flow patterns under microgravity conditions. Advances in Mechanics, 1999, 29(3): 369 382 (in Chinese) 
2 Aul RW, Olbricht WL. Stability of a thin annular film in pressure-driven, low-Reynolds-number flow through a capillary. J Fluid Mech, 1990, 215: 585 599

3 Lowe DC, Rezkallah KS. Flow regime identification in microgravity two-phase flows using void fraction signals. Int J Multiphase Flow, 1999, 25: 433 457

4 Lee J. Scaling analysis of two-phase gas-liquid flow pattern in microgravity. In: 31st Aerospace Sciences Meeting \& Exhibit, Reno, NV, January 11 14, 1993

5 Kim IW. Modelling of bubble and drop formation in flowing liquids in microgravity. [Ph.D thesis], Case Western Reserve University, 1992

6 Maxworthy T, Gnann C, Kürten M, et al. Experiments on the rise of air bubbles in clean viscous liquids. J Fluid Mech, 1996, 321: 421 441

7 Joseph DD, Bai R, Chen KP, et al. Core-annular flows. Annu Rev Fluid Mech, 1997, 29: 65 90

8 Jayawardena SS, Balakotaiah V, Witte LC. Flow pattern transition maps for microgravity twophase flows. AIChE J, 1997, 43: 1637 1640

9 Colin C, Fabre J, McQuillen J. Bubble and slug flow at microgravity conditions: state of knowledge and open questions. Chem Eng Comm, 1996, 141/142: 155 173

10 Zhao JF, Hu WR. Slug to annular flow transition of microgravity two-phase flow. Int $J$ Multiphase Flow, 2000, 26 (8): 1295 1304 